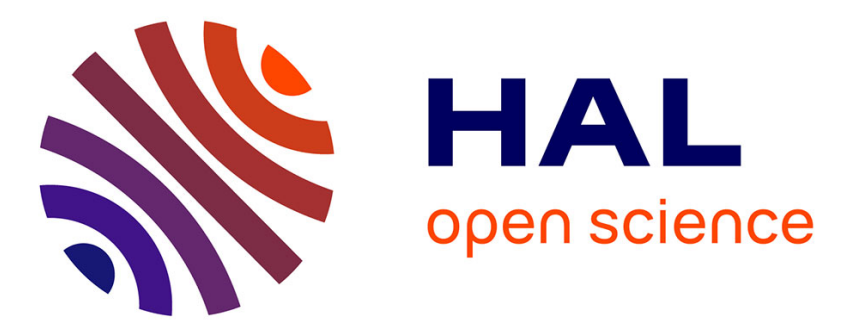

\title{
La question de la vulnérabilité et de l'adaptation de l'agriculture sahélienne au climat au sein du programme AMMA
}

Benjamin Sultan, Agali Alhassane, Bruno Barbier, Christian Baron, Marthe Bella-Medjo Tsogo, Alexis Berg, Michael Dingkuhn, Jeanne Fortilus, Mamoutou Kouressy, Antoine Leblois, et al.

\section{To cite this version:}

Benjamin Sultan, Agali Alhassane, Bruno Barbier, Christian Baron, Marthe Bella-Medjo Tsogo, et al.. La question de la vulnérabilité et de l'adaptation de l'agriculture sahélienne au climat au sein du programme AMMA. La Météorologie, 2012, Spécial AMMA, pp.64-72. hal-02873303

\section{HAL Id: hal-02873303 \\ https://hal.science/hal-02873303}

Submitted on 4 Jun 2021

HAL is a multi-disciplinary open access archive for the deposit and dissemination of scientific research documents, whether they are published or not. The documents may come from teaching and research institutions in France or abroad, or from public or private research centers.
L'archive ouverte pluridisciplinaire HAL, est destinée au dépôt et à la diffusion de documents scientifiques de niveau recherche, publiés ou non, émanant des établissements d'enseignement et de recherche français ou étrangers, des laboratoires publics ou privés. 


\title{
La question de la vulnérabilité et de l'adaptation de l'agriculture sahélienne au climat au sein du programme AMMA
}

\author{
Benjamin Sultan, Agali Alhassane, Bruno Barbier, Christian Baron, \\ Marthe Bella-Medjo Tsogo, Alexis Berg, Michael Dingkuhn, \\ Jeanne Fortilus, Mamoutou Kouressy, Antoine Leblois, \\ Romain Marteau, Bertrand Muller, Pascal Oettli, Philippe Quirion, \\ Philippe Roudier, Seydou B. Traoré et Michel Vaksmann
}

Les populations rurales d'Afrique soudano-sahélienne sont particulièrement exposées aux aléas climatiques et menacées par les effets attendus du changement climatique. Dans ce contexte, comprendre et anticiper les fluctuations climatiques ainsi que leurs conséquences sur l'agriculture constituent des enjeux majeurs pour le développement économique et la sécurité alimentaire en Afrique soudano-sahélienne. L'enjeu est de répondre au besoin sociétal de développer des stratégies pour réduire les impacts socio-économiques de la variabilité et du changement climatiques. Le programme AMMA, à travers la mobilisation multidisciplinaire qu'il suscite (réunissant notamment climatologues, hydrologues, agronomes, économistes autour d'objectifs communs) est un cadre idéal pour amorcer un réel dialogue entre les communautés des scientifiques et des utilisateurs pour répondre aux besoins prioritaires des populations. La première phase du projet a permis de réelles avancées sur trois objectifs majeurs : (i) mieux comprendre et quantifier les liens entre le climat et l'agriculture, (ii) quantifier la vulnérabilité de l'agriculture au changement climatique et (iii) s'adapter aux fluctuations et aux changements du climat. Cette synthèse illustrera, à partir de résultats issus de la première phase du programme AMMA, les avancées réalisées sur chacun de ces trois objectifs.

es populations rurales d'Afrique soudano-sahélienne sont particulièrement exposées aux aléas climatiques (Oram 1985 ; Hansen 2002 ; Barbier et al. 2009). Leur économie et leur sécurité alimentaire sont étroitement dépendantes de l'agriculture pluviale qui représente près de $93 \%$ des terres cultivées. Rappelons que $80 \%$ des céréales consommées en Afrique sub-saharienne proviennent de ce type de production. Le secteur agricole emploie $70 \%$ de la totalité de la main d'œuvre (FAO, 2003) et représente entre 15 et $20 \%$ du PIB. Outre cette dépendance, la croissance rapide de ces populations et un accès réduit aux adaptations technologiques (mécanisation, engrais, irrigation) constituent des facteurs aggravant des impacts socioéconomiques du climat (PNUD, 2004). Cette situation de vulnérabilité s'illustre par le fait que depuis les années 1970, les variations du climat, en aggravant fortement les conséquences de crises politiques, ont contribué aux plus grandes famines ayant nécessité un recours à l'aide alimentaire internationale (Dilley et al., 2005). Dans ce contexte, comprendre et anticiper les fluctuations climatiques ainsi que leurs conséquences sur l'agriculture constituent un enjeu majeur pour le développement économique et la sécurité alimentaire en Afrique sub-saharienne.
À ces fluctuations climatiques récentes s'ajoutent les conséquences attendues du changement climatique. Le quatrième rapport (AR4) du Groupe d'experts intergouvernemental sur l'évolution du climat (GIEC), publié en 2007, a en effet alerté la communauté internationale sur l' augmentation de la température partout dans le monde ainsi que sur la probable augmentation de la fréquence et de l'intensité des aléas météorologiques majeurs comme les sécheresses, en citant l'Afrique comme le continent le plus vulnérable aux changements climatiques. Les impacts de ces changements sur l'agriculture constituent une contrainte supplémentaire sur un système de production déjà vulnérable à la variabilité climatique actuelle et confronté à une croissance démographique très rapide.

Dans ce contexte, il est apparu essentiel que les progrès en termes de compréhension de la mousson africaine visés par le programme de recherche AMMA (Janicot et al., dans ce numéro, p. 2-8) puissent contribuer à répondre au besoin sociétal de développer des stratégies pour réduire les impacts socioéconomiques de la variabilité et du changement climatiques. Le programme AMMA, à travers la mobilisation multidisciplinaire qu'il suscite (réunissant notamment climatologues, 


\section{Abstract}

Vulnerability and adaptation of agriculture to climate variability and change in the Sahel: results from the AMIMA program

Rural populations in SoudanoSahelian Africa are known to be particularly vulnerable to climate change due to a combination of naturally high levels of climate variability, high reliance on climate sensitive activities such as rain-fed agriculture and limited economic and institutional capacity to cope with and adapt to climate variability and change. Urgent actions are required to tackle the issues raised by climate change in Soudano-Sahelian Africa and these actions need to be supported by the best knowledge available. The AMMA program is attempting to revitalize research in Africa in this field through an integrated interdisciplinary framework that aims to increase our understanding of the problem and support decision making for the future. The first phase of AMMA tackled three major issues: (i) increase our understanding of the relationships between climate and agriculture (ii) quantify the vulnerability of agriculture productivity to climate change and (iii) explore pathways to cope with climate variability and change. This synthesis will show results from the first phase of the AMMA program to illustrate progress in each of these three issues.
(1) FAOSTAT: Food and Agriculture Organization Statistical databases.

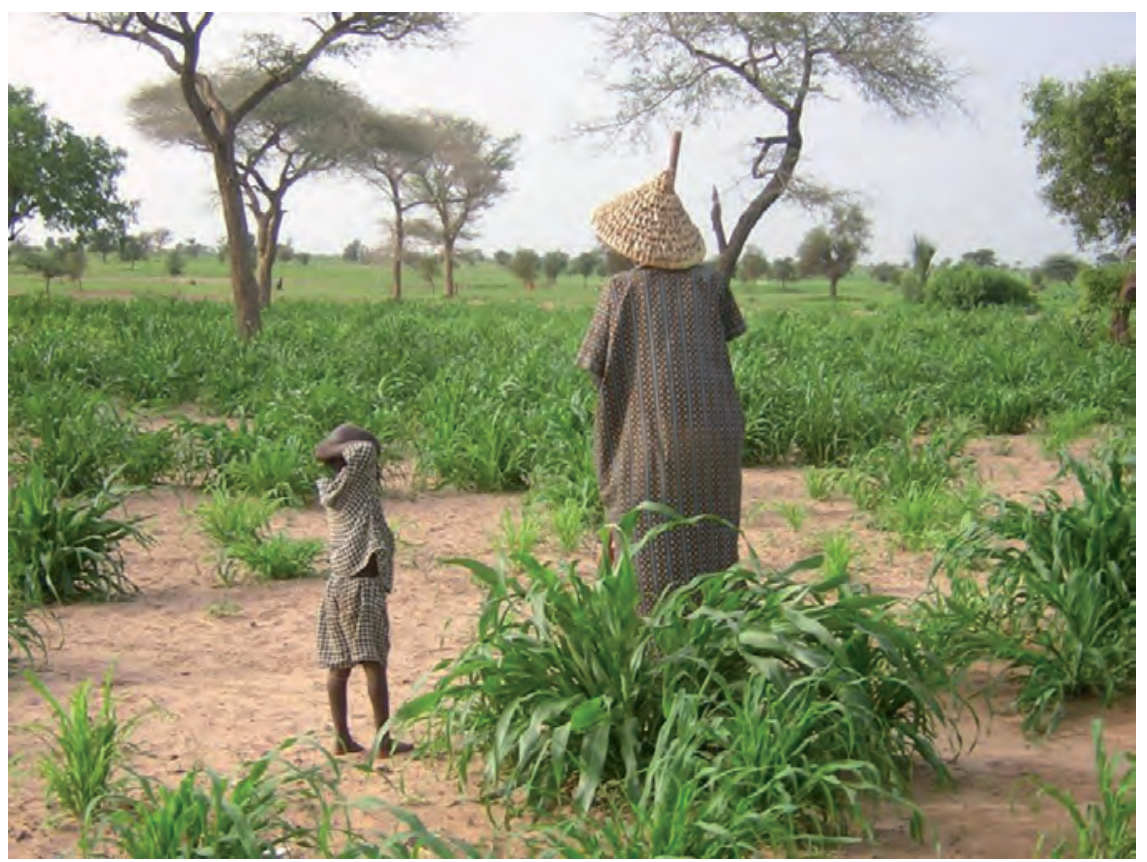

Un champ de cultures vivrières au Sénégal. (@ Bertrand Muller)

hydrologues, agronomes, économistes autour d'objectifs communs) est un cadre idéal pour amorcer un réel dialogue entre les communautés des scientifiques et des utilisateurs pour répondre aux besoins prioritaires des populations. La première phase du projet a permis de réelles avancées sur trois objectifs majeurs :

- mieux comprendre et quantifier les liens entre le climat et l'agriculture : l'information climatique seule n'est pas suffisante pour quantifier les conséquences des fluctuations ou des changements climatiques sur les ressources agricoles. Il est nécessaire de s'appuyer sur des systèmes d'observations et des outils de modélisation appropriés pour traduire cette information climatique en termes de risques ou de ressources pour le secteur agricole ; - quantifier la vulnérabilité de l'agriculture au changement climatique : même s'il existe encore de nombreuses incertitudes sur les conséquences du réchauffement global sur le climat africain (Fontaine et al., dans ce numéro p. 41-48), un tel bouleversement aura assurément des répercussions sur les ressources hydriques et sur la production agricole (CGIAR, 2009). Il apparaît donc crucial de pouvoir fournir une image plus précise de l'évolution attendue du potentiel de production agricole en Afrique de l'Ouest dans le contexte du réchauffement climatique ;

- s'adapter aux fluctuations et aux changements du climat : l'enjeu est tel que malgré l'incertitude sur les projections climatiques, il faut d'ores et déjà réfléchir aux options d'adaptation pour réduire la vulnérabilité de la population africaine à la variabilité et aux changements climatiques, et augmenter la sécurité alimentaire. C'est là que l'utilisation de l'information climatique pour orienter la stratégie agricole peut faire la différence, non seulement pour prévenir et minimiser les conséquences des aléas climatiques, mais aussi pour offrir des opportunités de capitaliser sur un climat plus favorable.

Cette synthèse illustrera, à partir de résultats issus de la première phase du programme AMMA, les avancées réalisées sur chacun de ces trois objectifs.

\section{Mieux comprendre et quantifier les liens entre le climat et l'agriculture}

Pour améliorer la compréhension des liens entre les systèmes agricoles au Sahel et le climat, le programme AMMA a mis en place des réseaux de mesures spécifiques en Afrique et s'est appuyé sur des outils de modélisation pour simuler la réponse des cultures vivrières comme le mil et le sorgho (64\% de la production céréalière d'Afrique de l'Ouest et $95 \%$ au Sahel d'après les données FAOSTAT ${ }^{(1)}$ en 2000) aux variations climatiques. 


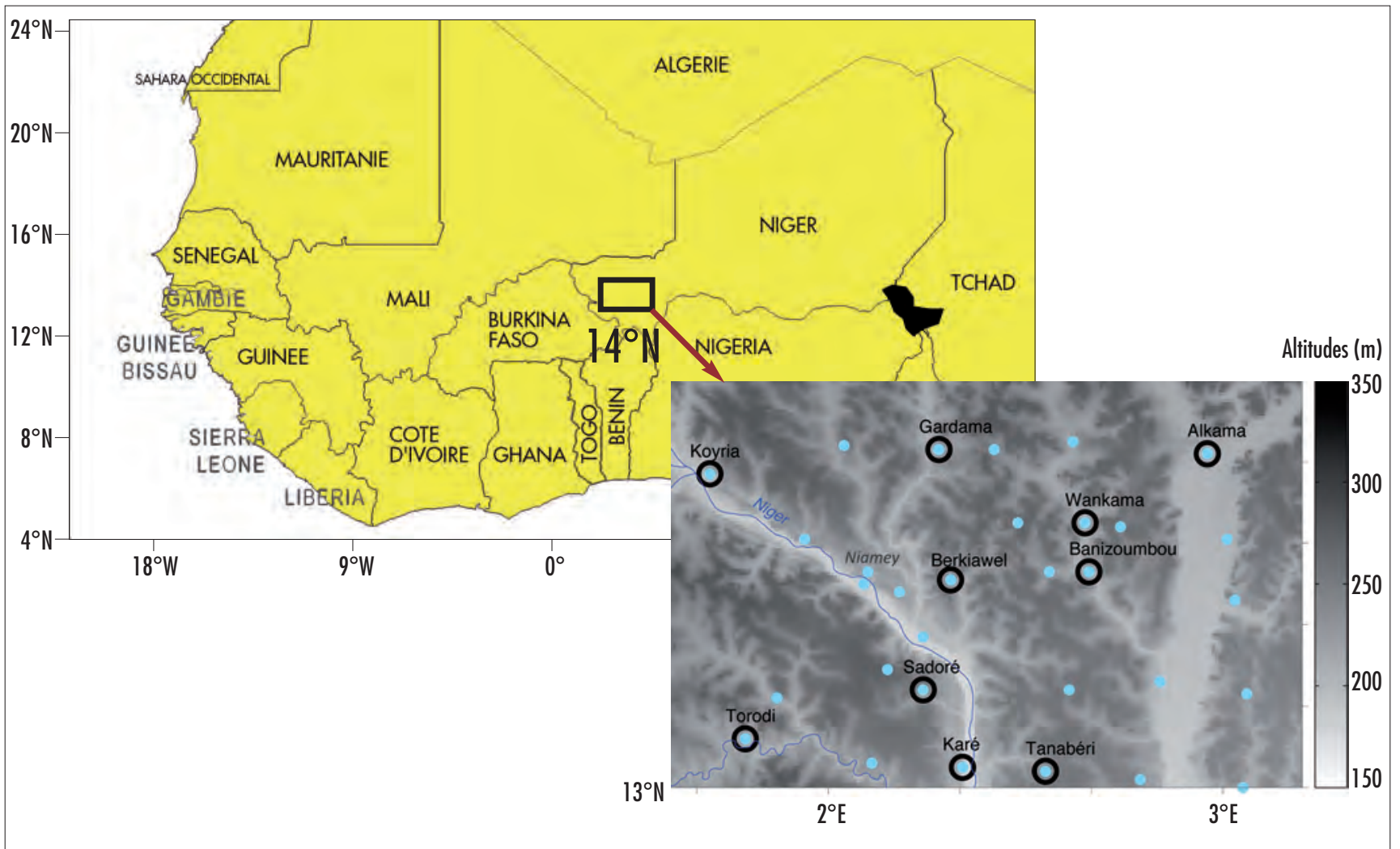

Figure 1 - Carte de l'Afrique de l'Ouest et localisation des 30 pluviographes du site de mésoéchelle AMMA-CATCH au Niger. Les cercles noirs correspondent aux 10 villages où sont menées depuis 2004 des enquêtes agronomiques. Tiré de Marteau et al. (2011).

\section{L'observation des systèmes de culture et de leur climat}

Le cour des actions d'AMMA portant sur l'agriculture est la mise en œuvre d'enquêtes agronomiques pour décrire les systèmes de culture à l'échelle de la parcelle agricole (Traoré et al., 2011) de manière à quantifier le rôle du climat sur les pratiques (dates de semis, resemis) et sur la productivité agricole (biomasse, rendement). Ces enquêtes ont d'abord été initiées en 2004 au Niger, sur le site de l'observatoire géophysique EPSATNiger constitué de 30 stations pluviographiques réparties sur un degré carré $(120 \times 160 \mathrm{~km})$ autour de Niamey. Sur ces 30 stations, 10 villages ont été retenus de façon à couvrir au mieux l'espace de l'observatoire (figure 1; Marteau et al., 2011). Pour chacun de ces 10 villages, on dispose d'observations sur 30 parcelles sur la culture du mil (seule céréale cultivée sur ce site) avec en particulier les dates semis/resemis, les cultivars sélectionnés, la densité de semis, l'apport d'intrants (quantité et nature, engrais, produits phytosanitaires), les dates des différentes phases phénologiques, les dégâts subis au cours du cycle (ravageurs, mauvaises herbes), la nature et la profondeur des sols, la préparation du sol (scarifiage, sarclage, labour) et les rendements en grains à la récolte. Les parcelles suivies sont situées à moins de deux kilomètres du pluviographe installé sur chacun des sites dans le cadre de l'observatoire AMMA-CATCH Niger. La répétitivité temporelle (tous les ans depuis 2004) et spatiale (30 parcelles par village et 10 villages) de ces enquêtes et son association avec l'observatoire géophysique EPSAT-Niger en font un site unique en Afrique de l'Ouest pour étudier les liens entre le climat et le rendement du mil à différentes échelles. À partir de 2006, un autre observatoire, composé de 3 sites le long d'un transect nord-sud (Diourbel, Kaffrine et Tambacounda) a été implanté au Sénégal selon un protocole similaire suivi par le CIRAD et le CERAAS. Cette action a été renforcée par l'arrivée de nouveaux partenaires africains au sein de l'extension du projet européen AMMA-TTC en 2007 et deux types d'activités ont été mises en place dans le cadre d'un réseau regroupant quatre pays de l'Afrique de l'Ouest (INERA au Burkina-Faso ; IER, U. Bamako et DMN au Mali ; UAM et INRAN au Niger ; ISRA, UCAD, DMN et CERAAS au Sénégal) :

- des enquêtes en milieu paysan visant à étudier la variabilité des rendements et ses déterminants au sein de petites régions agricoles ;

- et des essais agronomiques en stations expérimentales pour étudier les caractéristiques de croissance des variétés paysannes (longueur des phases et cycles, sensibilité à la température et au photopériodisme, biomasses aériennes, densité de semis).

\section{La modélisation agronomique}

Pour quantifier les liens entre le climat et l'agriculture, un prérequis est de construire un modèle qui permette de transcrire l'information climatique (températures et/ou précipitations, par exemple) en termes de variables agronomiques (rendements agricoles, biomasse). $\mathrm{Ce}$ type de modèle est particulièrement utile pour synthétiser les connaissances existantes sur les relations climat/plante, explorer des hypothèses de changements de climat ou de pratiques agricoles, identifier des variables clés sur lesquelles la recherche doit mettre l'accent et construire des scénarios pour le futur. Dans le cadre du programme AMMA, deux approches différentes ont été mises en œuvre visant à quantifier la réponse de la productivité agricole aux variations climatiques : la modélisation empirique qui consiste à construire un lien entre des variables à expliquer (comme le rendement par exemple) et des variables explicatives sans représenter explicitement les mécanimes agronomiques, et la modélisation mécaniste qui repose sur une décomposition en processus connus et sur une cohérence théorique. 
- La modélisation empirique. Ces modèles peuvent être construits à partir d'analyses statistiques reliant des observations agronomiques et climatiques (Schlenker et Lobell, 2010 ; Lobell et al., 2008). Cette approche, relativement simple et rapide à mettre en place, permet une focalisation directe sur des échelles ou des indicateurs pertinents pour les utilisateurs. La figure 2 illustre le type de relation statistique que l'on peut établir entre le climat et le rendement, à travers l'exemple de la culture du coton au Mali. Elle montre un lien entre le cumul pluviométrique annuel et le rendement du coton, avec des rendements d'autant plus forts que le cumul est important (Sultan et al., 2009). Cette relation, qui semble linéaire jusqu'à une valeur de cumul annuel inférieure à $900 \mathrm{~mm} / \mathrm{an}$, peut ainsi être utilisée pour prédire l'évolution du rendement du coton. Par ailleurs, une étude préliminaire conduite par Alhassane et Traoré (2011) montre qu'il est possible de développer une méthode de prévision saisonnière des rendements agricoles en Afrique de l'Ouest, sur la base de données historiques de températures de surface de la mer et des rendements de mil et de maïs observés.

- La modélisation mécaniste. Les modèles mécanistes permettent, pour une culture et/ou une variété de culture donnée, de simuler, généralement sur une base journalière, la croissance et le développement de la plante au cours de son cycle phénologique, afin de prédire la production de biomasse et le rendement à la fin de la saison de culture. Les campagnes de mesures, les expérimentations sur le terrain du programme AMMA ainsi que l'effort sur le développement numérique des modèles agronomiques ont permis de développer, de calibrer et de valider des modèles spécifiques pour simuler la réponse des céréales sèches (sorgho, mil et maïs) aux variations climatiques au Sahel. Des modèles comme SARRA-H (Système d'analyse régionale des risques agronomiques - version $\mathrm{H}$; Traoré et al., 2011 ; Dingkuhn et al., 2003) et ORCHIDEEMil (Berg et al., 2010) reproduisent bien la variabilité des rendements passés à l'échelle locale (Traoré et al., 2011) et régionale (figure 3 ) et ont l'avantage de représenter des relations non linéaires entre les variables climatiques et le rendement du mil (figure 3 ; Baron et al., 2005). Ces modèles peuvent être utilisés aussi bien dans le contexte de l'alerte précoce que pour des études exploratoires sur les impacts du changement climatique (Berg et al., 2012 ; Oettli et al., 2011 ; Alhassane et al., 2012).

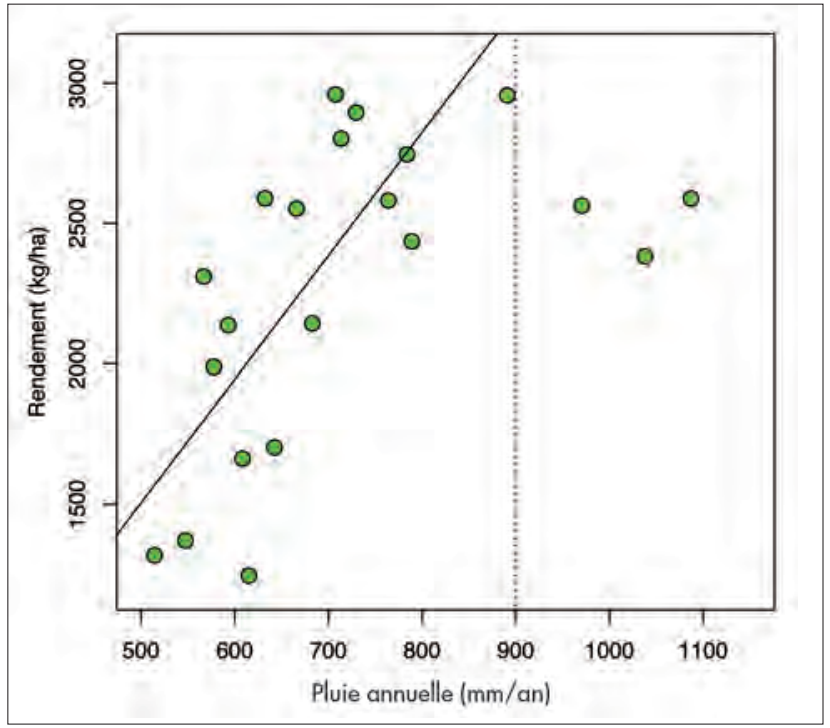

$\angle$ Figure 2 - Relation entre e rendement du coton au Mali et la pluie annuelle. Le rendement du coton est mesuré dans la station expérimentale de Koutiala par l'école supérieure européenne d'Ingénierie de I'espace rural (IER) et le SFCRJ, pour la période 1965-1990 (1968, 1972 et 1975 sont manquantes). La pluie (mm/an) est mesurée à la station de N'Tarla. La relation entre la pluie annuelle et le rendement du coton est linéaire lorsque le cumul pluviométrique annuel est inférieur à $900 \mathrm{~mm} / \mathrm{an}$ (trait dis continu). Tiré de Sultan et al. (2009).

V Figure 3 - Haut : Schéma de fonctionnement du bilan carboné et de la phénologie du modèle SARRA-H. Milieu : Rendements observés (noir) et simulés par SARRA-H (rouge) des céréales (mil et sorgho) à l'échelle de I'Afrique de l'Ouest sur la période 1961-1990. Les rendements sont présentés en anomalies standardisées et une tendance linéaire depuis le début de la période a été retirée.

Bas gauche : Relations entre le rendement du mil simulé par SARRA-H et la pluviométrie cumulée sur le cycle (mm/cycle) de culture pour 30 stations en Afrique de l'Ouest sur la période 1950-1980.

Bas droite : Idem mais avec le rayonnement global (Watt/cycle) en abscisse. Tiré de Baron et al. (2005).

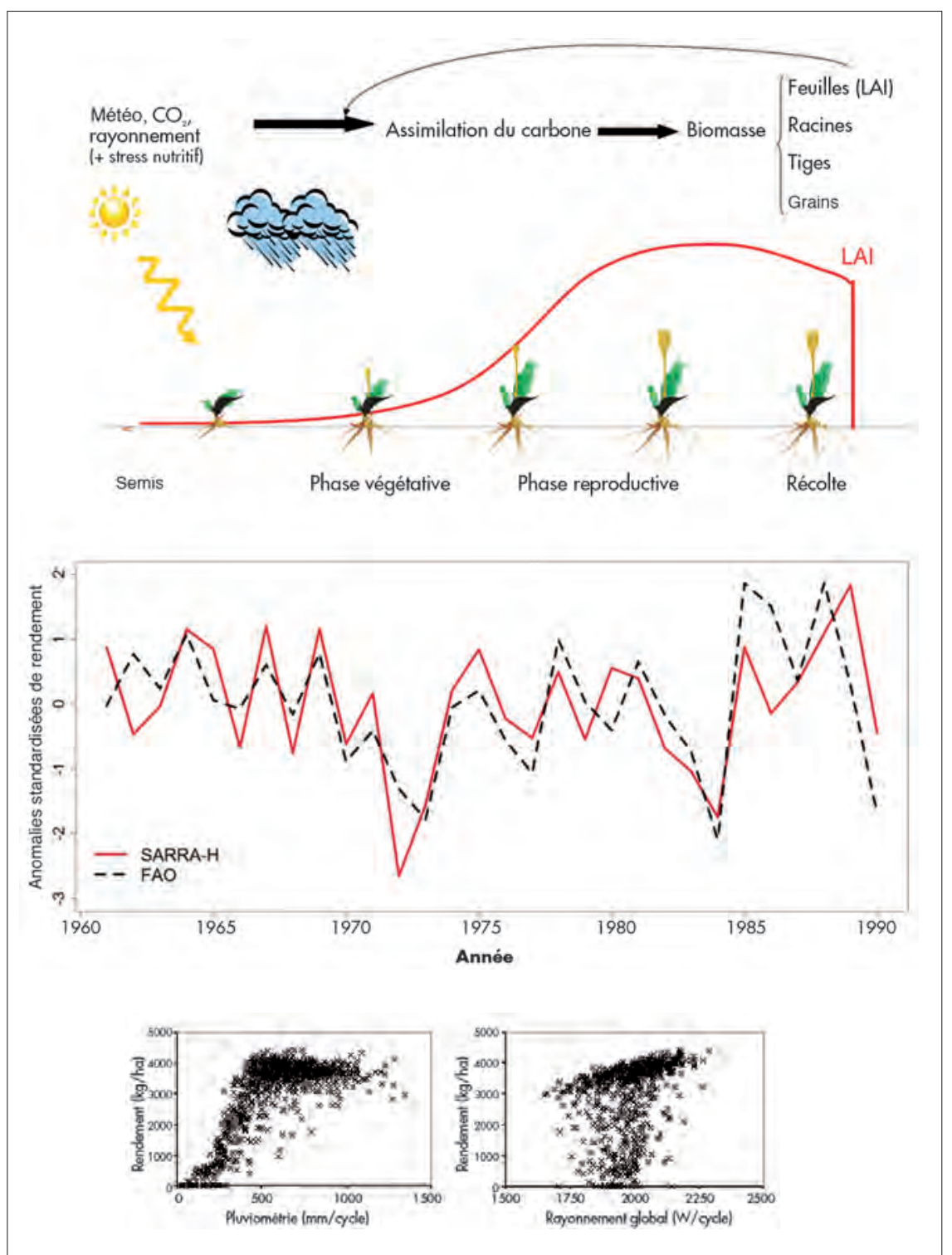




\section{Quantifier}

\section{la vulnérabilité de l'agriculture au changement climatique}

Compte tenu de l'extrême vulnérabilité du secteur agricole de l'Afrique subsaharienne aux aléas climatiques, il est crucial de pouvoir réaliser des projections fiables du devenir de la production agricole en réponse aux changements du climat. Cependant, cette tâche reste encore difficile à réaliser du fait des fortes incertitudes sur les projections régionales du changement climatique, sur la réponse des plantes aux changements environnementaux (pluie, températures, concentration de $\mathrm{CO}_{2}$ dans l'atmosphère), sur le couplage des modèles agronomiques et climatiques et sur la façon dont les systèmes agricoles vont s'adapter progressivement aux changements environnementaux (Challinor et al., 2007). L'objectif a donc été :

- de faire un bilan objectif de la littérature sur les effets du changement climatique sur l'agriculture en Afrique de l'Ouest ;

- et de comprendre les déterminants de cette réponse.

\section{Une baisse des rendements agricoles sous l'effet du changement climatique}

Il existe de nombreux articles et rapports faisant état de projections futures des rendements en Afrique subsaharienne en réponse aux changements environnementaux (Challinor et al., 2007). Cependant, ces documents portent tous sur un pays ou un groupe de pays en particulier, mettent l'accent sur une culture ou sur une variété spécifique et utilisent des méthodologies différentes (modèle empirique ou mécaniste pour simuler les rendements, différentes méthodes de régionalisation, différents modèles ou scénarios climatiques, prise en compte de l'effet du $\mathrm{CO}_{2}$ ). Il est donc assez difficile de se faire une idée d'ensemble de l'impact du changement climatique sur l'agriculture en Afrique et surtout des incertitudes dont sont assorties ces projections. Roudier et al. (2011a) ont procédé à une méta-analyse des résultats de la littérature en compilant les résultats issus de 16 publications récentes sur le sujet pour constituer une base de données de rendement pour le futur. La figure $4 a$ montre que le signe du changement relatif de rendement entre le climat présent et le climat futur est dans la plupart des cas négatif avec une baisse de l'ordre de $10 \%$ par rapport au présent. $\mathrm{Ce}$ chiffre est néanmoins assorti d'une incertitude forte puisque les distributions des réponses sont très étalées et varient de $-40 \%$ à $+80 \%$, selon les cas. La prise en compte de la concentration atmosphérique en $\mathrm{CO}_{2}$, qui a un effet fertilisant sur la plante, quoiqu'encore mal compris et mal représenté par les modèles (Long et al., 2006 ; Tubiello et al., 2007 ; Ainsworth et al., 2008), a un effet d'atténuation sur l'effet négatif du changement climatique bien que les impacts combinés des changements environnementaux (climat et $\mathrm{CO}_{2}$ ) restent globalement négatifs.

Il est également intéressant de constater que même si la dispersion des résultats issus de l'utilisation de modèles agronomiques mécanistes est plus forte (ce qui est logique car ces modèles sont
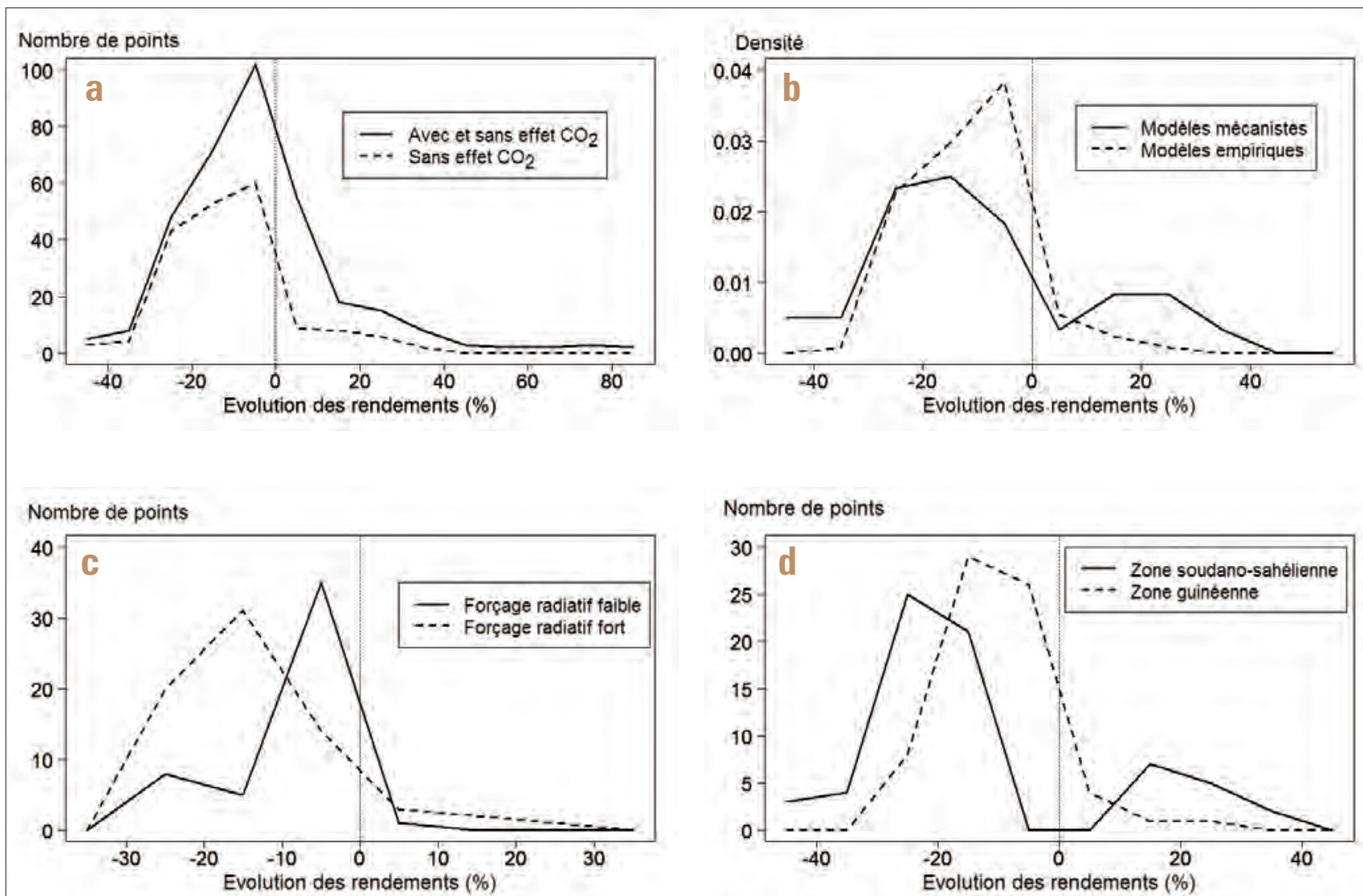

Figure 4 - (a) Distribution des changements relatifs de rendement en Afrique de l'Ouest entre le climat présent et le climat futur pour 16 études récemment publiées (trait continu). La courbe discontinue représente la même distribution mais uniquement pour les études ne considérant pas l'effet du $\mathrm{CO}_{2}$ sur la plante.

(b) Comme (a) mais en distinguant les résultats basés sur des modèles mécanistes (trait continu) de ceux basés sur les modèles empiriques (trait discontinu). (c) Comme (a) mais en distinguant les résultats selon que le forçage radiatif est relativement faible (trait continu) ou fort (trait discontinu).

(d) Comme (a) mais en séparant les résultats sur les pays soudano-sahéliens (trait continu) de ceux sur les pays guinéens (trait discontinu). Tiré de Roudier et al. (2011a). 
plus riches que les modèles statistiques), le signe du changement de rendement qu'ils prévoient est le même que pour les modèles empiriques (figure $4 \mathrm{~b}$ ). De plus, la figure $4 \mathrm{c}$ montre que plus la concentration de $\mathrm{CO}_{2}$ dans l'atmosphère considérée dans ces études est élevée (horizons temporels lointains, scénarios économiques avec de fortes émissions comme le scénario A2), plus l'impact négatif attendu sur les rendements est fort. Cette constatation donne un sens particulièrement important à la prise en compte de la réduction des émissions de $\mathrm{CO}_{2}$ par les mesures d'atténuation qui peuvent limiter les impacts sur l'agriculture en Afrique de l'Ouest. Enfin, la figure 4d montre un impact différentiel du changement climatique selon la région de l'Afrique de l'Ouest, les pays sahéliens étant plus durement touchés que les pays guinéens.

\section{Le rôle majeur de la température}

Pour comprendre les déterminants de la réponse des rendements agricoles au changement climatique, on utilise un modèle agronomique pour construire des projections futures du mil en Afrique de l'Ouest à partir des scénarios de changement climatique issus des modèles de climat du GIEC (rapport AR4). Berg et al. (2012) ont forcé le modèle agronomique ORCHIDEEMil (Berg et al., 2010) par les sorties d'un sous-ensemble des modèles climatiques du GIEC AR4. Des simulations transitoires ont été réalisées sur la période 1961-2000 (8 modèles) et sur le $\mathrm{XXI}^{\mathrm{e}}$ siècle avec les scénarios A1B ( 7 modèles) et A2 (5 modèles). Le choix des modèles s'est fait sur la base de la disponibilité des variables climatiques nécessaires pour forcer ORCHIDEE-Mil. Les simulations ont été réalisées avec et sans augmentation de la concentration du $\mathrm{CO}_{2}$ dans l'atmosphère de sorte que l'on a pu quantifier son effet de fertilisation sur les changements futurs des rendements. Le modèle ayant montré une bonne qualité de prédiction des rendements du mil en Afrique et en Inde (Berg 2011), les simulations ont été réalisées sur une fenêtre spatiale englobant l'Afrique et le subcontinent indien. Du fait des biais importants des modèles de climat, en particulier dans la distribution spatiale des précipitations en Afrique, il est difficile de conduire une analyse des impacts sur une base géographique. Par exemple, s'il ne pleut pas suffisamment au Sahel, comme c'est le cas dans le modèle climatique de l'Institut PierreSimon Laplace (IPSL) notamment, le modèle agronomique ne simulera que des très faibles valeurs de rendement, voire un rendement nul, et le changement dans le futur que donnera le modèle agronomique n'aura vraisemblablement que peu de sens. Berg et al. (2012) se sont donc orientés vers l'analyse des rendements simulés au sein de zones climatiques homogènes pour éliminer les incertitudes et les erreurs liées aux biais géographiques des modèles. Une version simplifiée de la classification de Köppen a été appliquée sur les sorties de chaque modèle de climat pour distinguer quatre zones climatiques homogènes : une zone équatoriale humide toute l'année, une zone équatoriale à saison sèche, une zone aride et une zone tempérée (figure 5). Les pixels de chaque classe diffèrent géographiquement selon les modèles mais partagent globalement les mêmes conditions climatiques.
On note une réponse très cohérente du rendement simulé entre les différents modèles de climat qui s'accordent tous à montrer une baisse des rendements dans trois des quatre grandes régions considérées (zone équatoriale humide, zone équatoriale à saison sèche et une zone tempérée ; figure 5). Ce signal, robuste avec le scénario A2, est largement dû à l'augmentation des températures, cohérentes d'un modèle à l'autre, qui réduit la longueur de cycle de la plante (Berg et al., 2012). Ce déficit de rendement dans le futur reste le même lorsque l'on considère l'effet du $\mathrm{CO}_{2}$ sur la plante ${ }^{(1)}$. Ce dernier est très faible dans le modèle car la photosynthèse du mil en $\mathrm{C} 4$ n'est que très

(1) Les végétaux peuvent être classés en plusieurs catégories selon les premières molécules organiques formées lors de la fixation du $\mathrm{CO}_{2}$ au cours de la photosynthèse : C3 le premier produit est un composé à 3 atomes de carbone), C4 (le premier produit est un composé à 4 atomes de carbone), CAM (le premier composé peut être un composé à 3 ou à 4 atomes de carbone). [Ndlr]
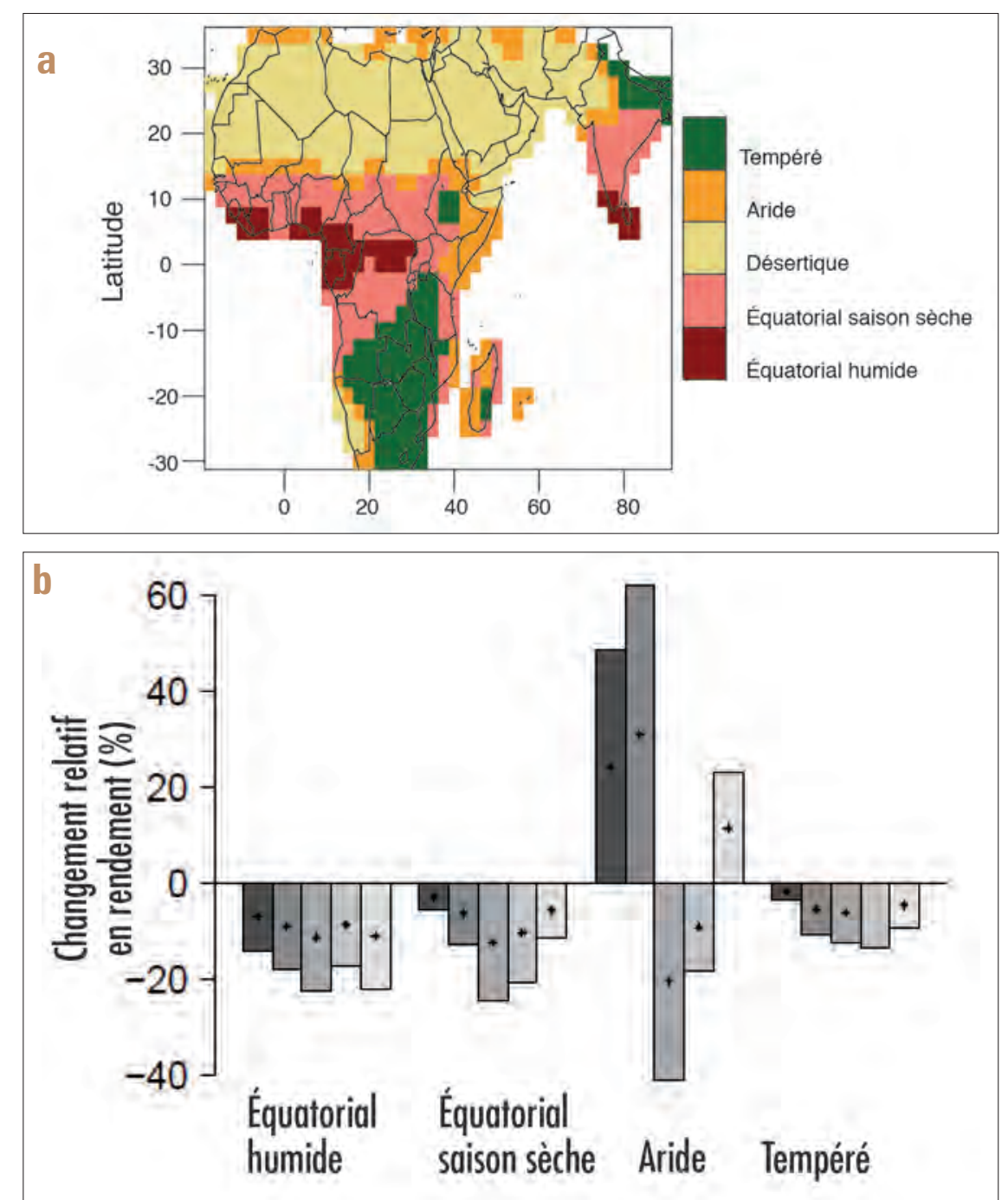

Figure 5 - (a) Localisation des zones climatiques de Köppen dans le modèle MIROC-MEDRES sur la période présente 1970-2000.

(b) Changement relatif du rendement (\%) entre le présent (1970-2000) et le futur (2070-2100), selon le scénario A2 pour les 5 modèles du GIEC AR4 considérés. Les points représentent les changements significatifs à $5 \%$. Tiré de Berg et al. (2012). 
peu sensible à l'effet fertilisant du $\mathrm{CO}_{2}$, contrairement à la photosynthèse en $\mathrm{C} 3$ (blé, coton, par exemple). Dans les zones arides où le rendement simulé est très sensible aux variations pluviométriques, aux franges du désert saharien notamment, la réponse en rendement est beaucoup plus divergente entre les modèles du fait de l'incohérence des projections de la pluviométrie annuelle. Dans ces zones, les modèles de climat dont les précipitations augmentent (diminuent) dans le futur conduisent à des baisses de rendements plus faibles (plus fortes). Si les effets des précipitations ont été dominants dans l'histoire récente, ces résultats montrent qu'il peut en être tout à fait différemment dans le futur avec un rôle important des températures dans l'évolution des rendements sur la majeure partie de l'Afrique, bien qu'une incertitude subsiste quant aux changements induits par les précipitations dans les régions les plus sensibles au stress hydrique. Ce rôle des températures sur l'évolution des rendements est confirmé par la littérature récente (Schlenker et Lobell, 2010 ; Salack, 2008).

Cependant, le déficit de rendement induit par l'augmentation des températures reste d'une amplitude assez faible, bien plus faible que celle que l'on peut observer dans la différence de rendement d'une parcelle à une autre sous l'effet de variations du type de sol ou d'apports d'intrants. De plus, si cette baisse de rendement provoquée par la réduction du cycle de culture sous un climat plus chaud peut être limitée par la sélection de variétés à cycle plus long, il subsiste encore de nombreuses incertitudes sur le futur des rendements agricoles en Afrique soudano-sahélienne. En effet, des modèles comme SARRA-H ou ORCHIDEE-Mil ne représentent pas l'effet de la température sur l'efficience de conversion de la lumière interceptée en biomasse ni celui des très hautes températures sur les grains et sur la mortalité des cultures. Ces deux effets peuvent aggraver le déficit de rendement induit par la réduction du cycle de culture.

\section{S'adapter aux fluctuations et aux changements du climat}

L'Afrique soudano-sahélienne ne peut pas attendre éternellement plus de précisions et de certitudes sur l'évolution du climat pour définir et appliquer des mesures d'adaptation, le climat d'aujourd'hui ayant déjà un impact très fort sur la production agricole. Pour lutter contre les aléas climatiques, qui sont loin d'être des phénomènes nouveaux en Afrique, les populations rurales ont privilégié depuis longtemps les pratiques agricoles les moins risquées (de Rouw, 2003) au détriment de techniques plus productives mais présentant des risques plus élevés (Affholder, 1997). Cependant, si ces stratégies sont efficaces pour assurer leur survie, elles limitent fortement le développement en entretenant un potentiel de production faible, même quand les conditions pluviométriques sont bonnes, ce qui maintient les populations rurales dans la pauvreté (CGIAR, 2009). L'utilisation de l'information climatique pour orienter la stratégie agricole est l'une des mesures possibles, non seulement pour prévenir et minimiser les conséquences des aléas climatiques, mais aussi pour offrir des opportunités de capitaliser sur un climat plus favorable.

\section{Date de démarrage \\ de la mousson et date de semis des cultures}

Plusieurs études récentes ont été menées pour identifier les besoins des agriculteurs en termes d'information climatique par le biais d'enquêtes et de questionnaires adressés aux utilisateurs (Ingram et al., 2002 ; Klopper et al., 2006). Elles ont toutes fait ressortir l'intérêt des agriculteurs pour l'information climatique comme aide à la prise de décision. Ingram et al. (2002), à travers

La récolte du coton au Mali. (C Mamoutou Kouressy)

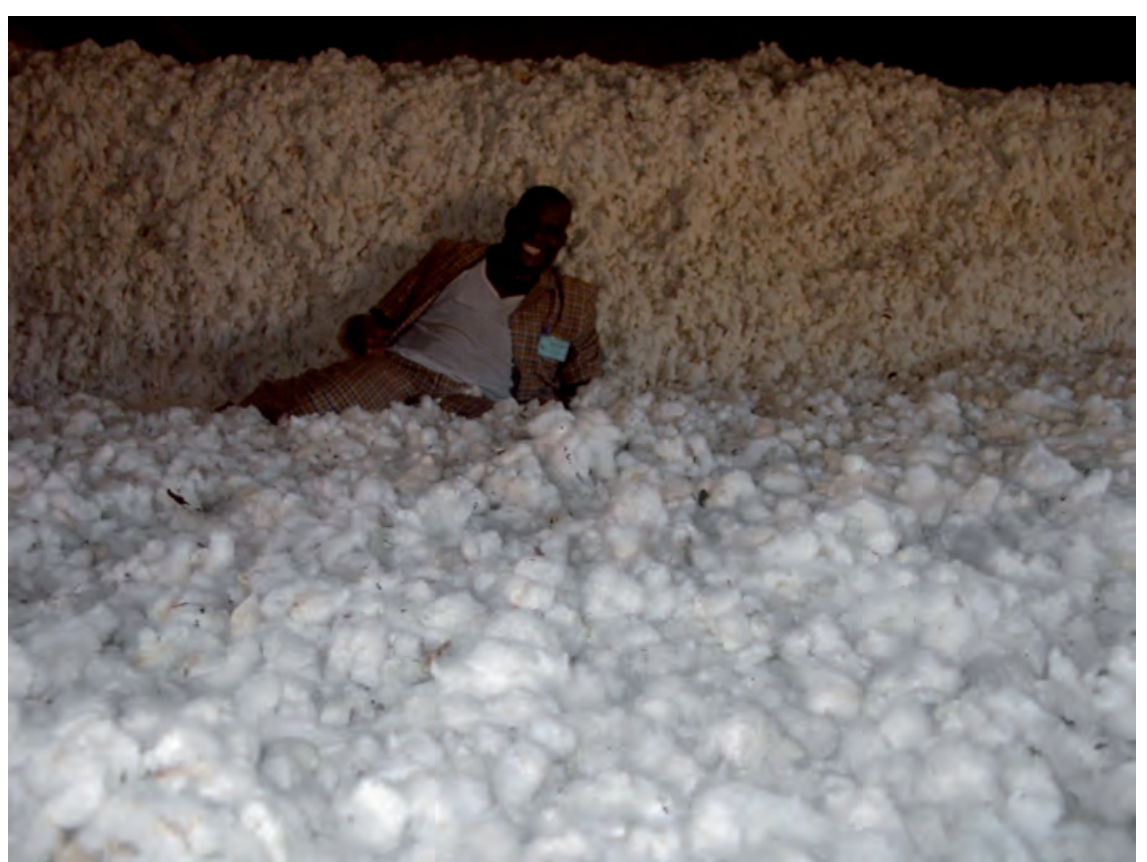

des enquêtes sur plusieurs catégories d'agriculteurs au Burkina Faso, ont essayé de classer les indicateurs climatiques en fonction de leur importance l'aide à la décision agricole. Ces indicayeux des agriculteurs burkinabés, sont : - les dates de démarrage et de fin de la saison des pluies ;

- la répartition des pluies au sein de la saison humide ;

- et le cumul saisonnier des pluies.

La date de démarrage des pluies est ainsi considérée comme l'indicateur climatique le plus important pour les agriculteurs puisqu'il conditionne la date de semis des cultures. Marteau et al. (2011) ont analysé la stratégie de semis des agriculteurs au Niger vis-à-vis de la pluviométrie, à partir des données recueillies en milieu paysan pour la période 2004-2009, dans 10 villages situés sur un domaine d'un degré carré au Niger. Ils ont montré que dans la majeure partie des cas, les paysans sèment le mil après une première pluie significative au printemps (généralement apportant plus de $10 \mathrm{~mm}$ en un jour), partant ainsi du postulat que cette première pluie marque le démarrage de la mousson. En l'absence d'information ou de prévision de la date de démarrage des pluies, les agriculteurs prennent ainsi le risque de perdre leurs semences si cette première pluie est provoquée par un événement isolé et se trouve suivie d'un épisode sec de plus de 7 jours après le semis. Marteau et al. (2011) ont ainsi montré que $22 \%$ des semis ont été perdus à cause de l'occurrence d'un perçue par les acteurs locaux pour teurs, classés par ordre d'intérêt aux 
épisode sec prolongé (supérieur à 7 jours) juste après le semis, autrement dit après un faux départ de la mousson. Si les agriculteurs connaissaient à l'avance la date de son démarrage, ils pourraient non seulement réduire les pertes de semences et de temps de travail liées aux semis ratés, mais aussi augmenter leurs rendements en calant mieux le cycle cultural à la saison des pluies, comme l'a montré l'étude de modélisation de Sultan et al. (2005a). Cependant, il faut préciser que pour répondre aux besoins des agriculteurs, la prévision de la date du démarrage de la mousson devrait atteindre une précision tant spatiale que temporelle qui est très loin d'être atteinte actuellement.

\section{Les bénéfices potentiels de l'utilisation de la prévision saisonnière}

En Afrique de l'Ouest, même s'il existe un système de prévision opérationnel qui fournit chaque année des prévisions, des mois à l'avance, sur la qualité de la saison des pluies à venir (le système PRESAO, Prévision saisonnière en Afrique de l'Ouest ; Hamatan et al., 2004 ; Ward et al., 2004), il n'existait aucune étude évaluant les bénéfices de la prévision saisonnière pour l'agriculture en Afrique sub-saharienne, ni même pour l'agriculture de subsistance en général. Actuellement, l'ensemble des évaluations est réalisé pour des cultures de rente (Meza et al., 2008). Pourtant l'évaluation des bénéfices de l'utilisation de la prévision saisonnière est nécessaire pour orienter la recherche là où les bénéfices sont les plus importants (pour une catégorie d'exploitants par exemple ou bien avec la prévision d'un indicateur climatique plutôt qu'un autre) et pour attirer les partenaires institutionnels et les investissements (Thornton, 2006 ; Meza et al., 2008). Sultan et al. (2010) et Roudier et al. (2011b) ont été parmi les premiers auteurs à avoir évalué les bénéfices de la prévision saisonnière en Afrique de l'Ouest, en couplant la modélisation climatique, économique et agronomique. En se basant sur un modèle bioéconomique calibré sur une exploitation agricole typique de Nioro du Rip, qui se trouve dans la région semi-aride du Sénégal, Sultan et al. (2010) ont mis en balance les bénéfices de l'utilisation de la prévision saisonnière par rapport aux pertes induites par des erreurs de prévision et montré que l'agriculteur peut tirer des bénéfices de la prévision saisonnière. Plus récemment, Roudier et al. (2011b) ont évalué les bénéfices de la prévision saisonnière pour la culture du mil au
Niger. Pour reproduire de manière fine les itinéraires techniques des paysans, les auteurs ont utilisé le modèle SARRA-H, capable de simuler la réponse en rendement (que l'on traduit ensuite en revenu pour les agriculteurs) au changement de stratégies (date de semis, niveau de fertilisation, longueur de cycle et type de variété cultivée). Ils ont mis en évidence un bénéfice de l'utilisation de la prévision quel que soit le type d'année (sèche, normale ou humide) considéré, avec une valeur maximale de la prévision en cas d'année humide (figure 6). Roudier et al. (2011b) se sont ensuite intéressés à faire évoluer la qualité de la prévision saisonnière afin de voir comment le progrès de la prévision climatique se traduit en termes de bénéfices pour les agriculteurs au Niger. Trois types de prévisions ont été considérés : la prévision imparfaite du cumul saisonnier (typiquement celle issue du système actuel PRESAO), la prévision parfaite du cumul saisonnier (typiquement si on améliore fortement le système PRESAO, sans changer les variables qu'il prévoit) et la prévision parfaite du cumul saisonnier avec en plus la prévision du début et de la fin de la saison des pluies (typiquement si on améliore le système PRESAO avec de nouvelles variables prévues). Il est intéressant de constater que l'augmentation la plus forte du bénéfice de la prévision ne se réalise pas avec l'amélioration du score du système existant (le passage de la prévision imparfaite à parfaite n'entraîne qu'une augmentation faible du bénéfice espéré dans la figure 6) mais plutôt grâce à l'introduction de la prévision de variables supplémentaires, comme le démarrage et la fin de la saison des pluies, qui permet un meilleur calage du cycle de culture (date de semis, longueur de cycle de la variété de mil utilisée) avec la saison humide. Ce résultat confirme l'importance de la prévision de la date de démarrage des pluies pour l'agriculture (Sultan et al., 2005a ; Marteau et al., 2011) et qui a été soulignée très justement par les agriculteurs sahéliens comme la priorité en terme d'indicateur climatique à être inclus dans les systèmes de prévision actuels (Ingram et al., 2002).

\section{Les assurances agricoles basées sur des indices climatiques}

La mise en place d'un système d'assurance agricole figure parmi les pistes possibles pour atténuer la vulnérabilité des agriculteurs aux fluctuations climatiques. Les assurances agricoles traditionnelles, basées sur la constatation d'une mauvaise récolte, sont actuellement peu développées dans les pays du Sud à cause de la difficulté d'évaluer les dommages et de l'asymétrie d'information entre le paysan et l'assureur. Depuis quelques années, des assurances basées sur des indices météorologiques ont été lancées à l'échelle de pilotes et même à plus grande échelle dans quelques pays en développement (Malawi, Ethiopie, Inde, Maroc et Mexique) mais pas encore en Afrique de l'Ouest (Leblois et Quirion, 2011). Pourtant, elles pourraient y atténuer les conséquences potentiellement dramatiques d'une mauvaise saison des pluies pour les paysans. Elles pourraient également faire évoluer les pratiques agricoles vers des pratiques plus risquées mais bénéfiques en moyenne : augmentation des intrants, expansion des cultures risquées mais en moyenne plus productives. Un des objectifs d'AMMA a donc été de montrer le potentiel de ce type d'assurances en Afrique de l'Ouest où elles n'ont

Figure 6 - Variations de revenus (\%) liées à l'utilisation de la prévision saisonnière pour la culture du mil au Nige selon que l'année est humide, normale, sèche et pour l'ensemble des années. Trois types de prévisions sont considérées : "Impft » correspond à la prévision catégorielle imparfaite du cumul saisonnier (typiquement celle issue de PRESA0), " Pft " est la même prévision mais sans erreur, et " $\mathrm{Pft}++$ " la prévision sans erreur avec en plus la prévision du début et de la fin de la saison des pluies. Tiré de Roudier et al. (2011b).

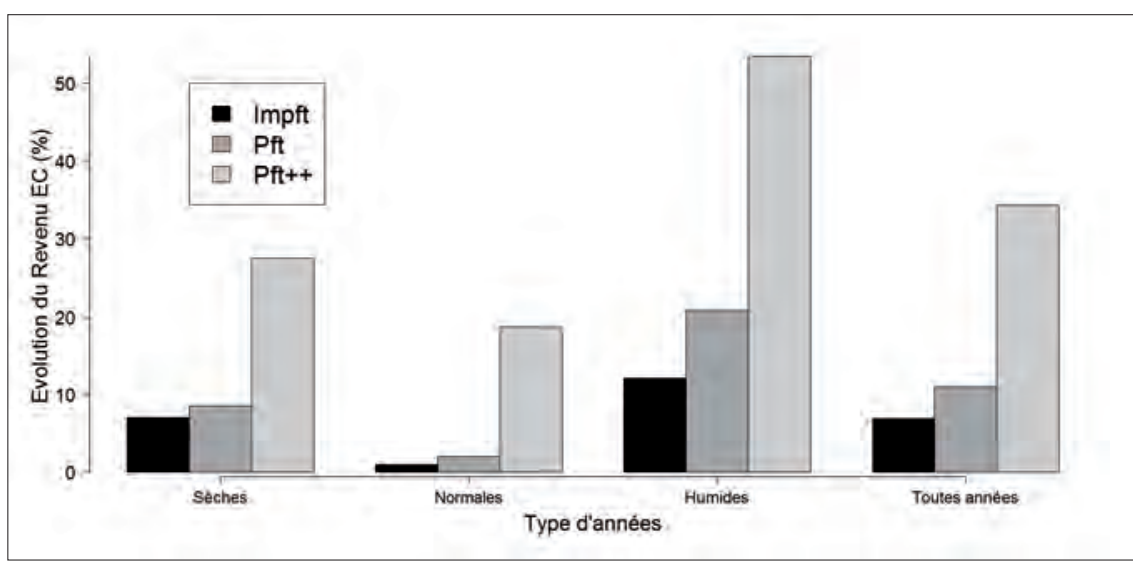


jamais été appliquées. À l'aide d'une base de données agricoles et pluviométriques détaillée sur le Burkina Faso et d'hypothèses simples sur la forme d'un contrat d'assurance, Berg et al. (2009) ont étudié la faisabilité potentielle et l'intérêt pour les agriculteurs de ce pays d'une assurance climatique à indice. Pour cela, ils ont considéré les résultats de plus de 3000 contrats simulés sur 30 provinces, 21 ans (1984-2004) et 5 cultures (coton, mil, sorgho, maïs et arachide). Le principe du contrat d'assurance consiste à trouver un indicateur qui fait le lien entre les fluctuations pluviométriques et le rendement des cultures, ici le cumul pluviométrique annuel, et à indemniser le producteur lorsque la valeur annuelle de cet indice est inférieure à un certain seuil (en cas de sécheresse avérée par exemple). Il est ressorti qu'un tel système d'assurance, même fondé sur un indice simple comme le cumul pluviométrique de la saison des pluies, peut être efficace d'un point de vue économique. Son efficacité est surtout déterminée par l'intensité du lien entre le climat et la culture (que l'on appelle le risque de base) mais surtout par la variance du rendement de la culture. Le maïs, dont le rendement est le plus variable d'une année sur l'autre, est la culture qui bénéficierait le plus de la mise en place d'un tel système d'assurance. D'autre part, les zones à faible pluviométrie annuelle au nord du Burkina-Faso pourraient également y trouver un bénéfice plus important du fait du lien plus fort entre les fluctuations climatiques et le rendement des cultures et des variations interannuelles importantes du rendement. Toutefois, de nombreux obstacles tendent encore à limiter leur mise en œuvre dans les pays en développement, en particulier l'intérêt limité des paysans pour des assurances payantes au regard de leur solvabilité réduite. Il semble aussi que l'intérêt de ce type d'outils de mutualisation réside dans sa complémentarité avec d'autres méthodes pour augmenter les rendements moyens. Il s'agit, par exemple, de l'utilisation d'intrants coûteux et souvent trop rares, ou encore des prévisions saisonnières, toujours imparfaites, pouvant alors augmenter le risque pris par les paysans lors de mauvaises saisons mal anticipées par ces dernières. Finalement, il sera important, pour favoriser leur développement futur, de trouver des outils innovants facilitant 1 'accès à cette mutualisation de moyenne ou de grande échelle et permettant d'augmenter la confiance des paysans dans ce genre d'outils et 1'implication des institutions locales existantes.

\section{Conclusion}

Anticiper les fluctuations et les changements climatiques est d'une importance primordiale pour l'agriculture en Afrique soudano-sahélienne. D'un côté les aléas climatiques peuvent être responsables de déficits de production et de graves crises alimentaires mais de l'autre la maîtrise et l'anticipation de la variabilité climatique peuvent être un premier pas vers une augmentation de la production et la concrétisation des objectifs du Millénaire visant à une plus grande sécurité alimentaire en Afrique.

L'étude des relations entre le climat et l'agriculture nécessite une approche pluridisciplinaire combinant notamment des données et des outils issus de l'agronomie (modèles empiriques ou mécanistes simulant la réponse du rendement aux variations climatiques), de la science du climat (modèles climatiques capables de réaliser des projections du climat de quelques mois à un siècle d'échéance) et de l'économie (scénarios d'évolution de la société, modèles bioéconomiques, adaptation). Certains de ces outils ont été développés, perfectionnés et validés dans le cadre de la première phase d'AMMA et permettront une pérennité de l'effort du programme, que ce soit à travers des applications opérationnelles en intégrant ces indicateurs au sein de systèmes d'alertes précoces ou à travers des études exploratoires sur les interactions entre le climat et l'agriculture dans les régions tropicales.

La quantification des impacts agronomiques du changement climatique nécessite la mise en place d'une méthodologie souvent complexe avec des outils tirés de l'économie (scénarios d'émission, modèles de décision des ménages agricoles), de la science du climat (modèles et projections climatiques), de l'agronomie (modèles agronomiques) et de la statistique (régionalisation et correction de biais), ayant chacun leur part d'erreur et leurs limites. Malgré ces incertitudes, il a pu être montré une baisse plausible de la productivité des céréales dans le futur sous l'effet du réchauffement climatique. Néanmoins, le défi à relever ne réside donc pas tant dans l'estimation déterministe et vraisemblablement inatteignable de l'évolution future des rendements mais dans la quantification, la hiérarchisation et la réduction des incertitudes associées aux projections des impacts du changement climatique. Le cadre des nouveaux projets internationaux CORDEX (projet d'intercomparaison de méthodes de régionalisation) et AGMIP (intercomparaison des modèles agronomiques) marquera très certainement un tournant vers une meilleure prise en compte de cette incertitude à travers des études coordonnées dédiées aux impacts du climat sur l'agriculture, qui étaient jusqu'alors menées de manière très isolée et fragmentée.

Cependant, il ne faut pas attendre plus de certitudes pour d'ores et déjà proposer des mesures d'adaptation qui soient à la fois scientifiquement pertinentes et socialement acceptables, le climat d'aujourd'hui ayant déjà un impact sur les ressources des populations rurales. L'utilisation de l'information et la prévision climatique pour orienter la stratégie agricole peut permettre dans bien des cas, illustrés dans le programme AMMA, d'augmenter les revenus et de réduire la vulnérabilité de l'agriculture traditionnelle. C'est aussi une forme d'adaptation « sans regret » au changement climatique car même si le climat ne change pas dans le futur, elle augmente la résilience aux aléas climatiques auxquels l'Afrique est fréquemment confrontée. Néanmoins, l'étude des vulnérabilités et de l'adaptation aux changements environnementaux nécessite d'aller bien au-delà de l'effort mené dans le cadre de la première phase et, en particulier, requiert un dialogue des sciences biophysiques (climat, hydrologie, agronomie) et des sciences humaines (démographie, histoire, anthropologie, économie). Cette approche pluridisciplinaire est cruciale lorsqu' on aborde la problématique de l'adaptation aux changements environnementaux où la réponse des sociétés sahéliennes est enchâssée dans des transformations sociales globales et où la variable climatique est loin d'être le seul et unique facteur de vulnérabilité. Les relations de l'homme à son environnement s'inscrivent en effet dans des configurations économiques, sociales et politiques complexes qu'il est nécessaire d'interroger et de confronter avec les phénomènes climatiques et environnementaux. La construction de ce dialogue pluridisciplinaire est le défi qui sera relevé dans la deuxième phase d'AMMA et mis en œuvre notamment dans le cadre des projets RIPIECSA, ESCAPE, ECLIS

\section{Remerciements}

Ce travail a été réalisé grâce aux financements du Groupement d'intérêt scientifique (GIS) « Climat-EnvironnementSociétés » dans le cadre du projet REGYNA, du Réseau de recherche sur le développement soutenable de la région Île-de-France (R2DS) et du programme AMMA (AMMA-IP, AMMA-API). 